Research Article

\title{
Preparation and Characteristic of PC/PLA/TPU Blends by Reactive Extrusion
}

\author{
Yueyun Zhou, ${ }^{1}$ Lifa Luo, ${ }^{2}$ Wenyong Liu, ${ }^{3}$ Guangsheng Zeng, ${ }^{3}$ and Yi Chen ${ }^{3}$ \\ ${ }^{1}$ Institute of Architecture and Urban Planning, Hunan University of Technology, Zhuzhou 412007, China \\ ${ }^{2}$ ZhengYe Packaging Limited Company of Zhongshan, Zhongshan, China \\ ${ }^{3}$ Key Laboratory of Advanced Materials and Technology for Packaging, Hunan University of Technology, Zhuzhou 412007, China \\ Correspondence should be addressed to Yi Chen; yiyue514@aliyun.com
}

Received 3 August 2015; Revised 12 October 2015; Accepted 26 October 2015

Academic Editor: Hossein Moayedi

Copyright (C) 2015 Yueyun Zhou et al. This is an open access article distributed under the Creative Commons Attribution License, which permits unrestricted use, distribution, and reproduction in any medium, provided the original work is properly cited.

\begin{abstract}
To overcome the poor toughness of PC/PLA blends due to the intrinsic properties of materials and poor compatibility, thermoplastic urethane (TPU) was added to PC/PLA blends as a toughener; meantime, catalyst di-n-butyltin oxide (DBTO) was also added for catalyzing transesterification of components in order to modify the compatibility of blends. The mechanical, thermal, and rheological properties of blends were investigated systematically. The results showed that the addition of TPU improves the toughness of PC/PLA blends significantly, with the increase of TPU, the elongation at break increases considerably, and the impact strength increases firstly and then falls, while the tensile strength decreases significantly and the blends exhibit a typical plastic fracture behavior. Meantime, TPU is conducive to the crystallinity of PLA in blends which is inhibited seriously by PC and damages the thermal stability of blends slightly. Moreover, the increased TPU makes the apparent viscosity of blends melt decrease due to the well melt fluidity of TPU; the melt is closer to the pseudoplasticity melt. Remarkably, the transesterification between the components improves the compatibility of blends significantly, and more uniform structure results in a higher crystallinity and better mechanical properties.
\end{abstract}

\section{Introduction}

In recent years, considerable attention has been paid to biodegradable polymers, mainly owing to increasing interest for preservation of environment and substitution for petrochemical polymers. Poly(lactic acid) (PLA), as one of the typical biodegradable polymers, has been studied and applied in many fields including tissue engineering $[1,2]$, drug delivery [3], and packing materials $[4,5]$. PLA has become a great alternative to traditional plastics as an environmentally friendly polymer due to its superior properties such as high strength, high stiffness, and resistance to fats and oil. However, significant brittleness, low viscosity, low thermal stability, high moisture sensitivity, and low solvents resistance are often insufficient for the applications a lot of regions [6]. Many researches have focused on the design and preparation of new materials with excellent properties; typically, blending biodegradable polymers with conventional polymers is considered a convenient and effective method. A lot of blends with PLA such as PLA/starch [7-9], PLA/ poly(vinyl alcohol) (PVA) [10], PLA/polycaprolactone (PCL) [11], PLA/montmorillonite [12], PLA/poly (butylene adipateco-terephthalate) (PBAT) [13], and PLA/rubber [14] have obtained in-depth research.

Recently, there is activity in blending PLA bioresins with engineering polymers to prepare alloy materials for using in durable products such as laptops, cellphones, and autoparts, which brings the biodegradablity character to the materials meanwhile keeps the excellent mechanical properties belonged to the engineering polymers. Some interesting systems were exploited; for example, Zhang et al. reported a PA/PLA blend with excellent toughness resulting from toughening by polyamide elastomer, and the shape memory effect was also found [15]. Polycarbonate (PC) is a typical engineering polymer which is commonly used in automotive, sheet glazing, medical appliance, packing, and some electrical 


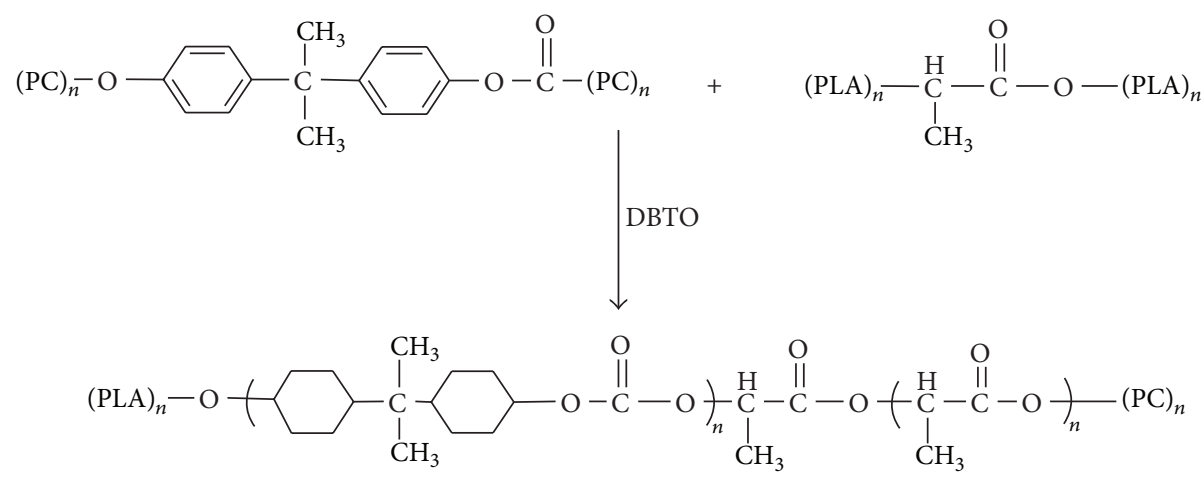

(a) Reaction between PC and PLA

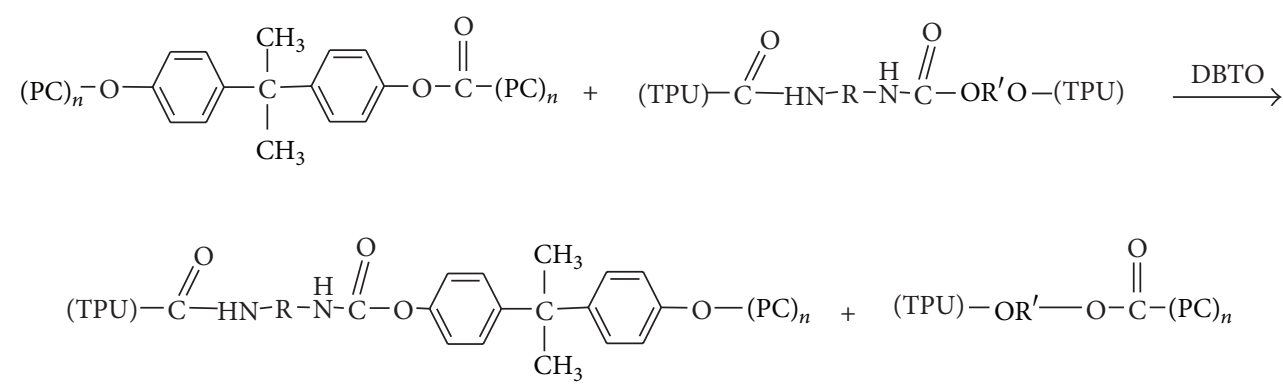

(b) Reaction between PC and TPU

SCHEME 1: The reaction equation of transesterification in blends. (a) Reaction between PC and PLA. (b) Reaction between PC and TPU.

applications because of its high tensile strength, good electrical properties, low coefficient of thermal expansion, clarity, dimensional stability, and high-heat deflection temperature. However, the PC resin as a synthetic plastic is hard to be rapidly decomposed which is considered as the main reason to cause the environmental pollution. Considering, comprehensively, incorporating PLA into PC is an effective method for preparing the alloy with excellent mechanical properties and biodegradability. Nevertheless, PC/PLA blends still exhibit significant brittleness just like the character of the pure PC and PLA resin; meantime, the deteriorated processability, appearance, and mechanical properties are also the great drawbacks of blends due to the poor compatibility between PC and PLA [15]. Finding a method to improve the compatibility and the toughness of PC/PLA blends is the key to the application of this kind of blends. In previous researches, different kinds of resins were incorporated into $\mathrm{PC}$ resin as flexibilizer for improving its notched impact resistance and rheological properties, such as acrylonitrilebutadiene-styrene (ABS) [16], ethylene vinyl acetate (EVA) [17], and thermoplastic urethane elastomers (TPU) [18] that were all proved effective. Among all of flexibilizers, TPU has attracted wide attention in many fields due to its excellent mechanical properties of high elasticity, great flexibility and toughness, and high resistance to tear, oxidation, and humidity, and it has been usually used instead of old polymers and other elastomers. Moreover, TPU has also been proved as an effective flexibilizer to improve the toughness of PLA [19]. Therefore, it is reasonable to believe that TPU would be an available flexibilizer in improving the performance of PC/PLA blends. Meantime, except for the choice of raw materials, for guaranteeing the excellent properties of blends with biopolymers, many factors need to be considered and controlled, and many methods revolved in various aspect of materials preparation were used, for example, designing an effective and controllable processing to adjust the molecular structure and properties of blends [20-22], adding nucleant agents or other materials in process to induce the crystallinity [23], forming chain extension-branching of biopolymers in processing through in situ reaction [24], and introducing the reaction between biopolymers and other functional polymers in processing [25]. In the systems with several components, the most important factor affecting the properties of blends is the compatibility of different components in blends. To solve this problem, the molecular structure of all components should be considered carefully. If the ester groups exist in all components, such as PLA, PC, and TPU, which could happen in the transesterification catalyzed by special catalyst, there is no doubt that the transesterification that happened between the components could make the blend tend to be whole thereby resulting in the improved compatibility of blends significantly.

In this study, PC/PLA/TPU blends with various ratio of raw materials were prepared by melt blending, an effective catalyst di-n-butyltin oxide (DBTO) was also added to the blends for catalyzing the transesterification between PC, TPU, and PLA in order to improve the compatibility of blends, and the mechanism was shown in Scheme 1. The effect of blends composition and transesterification on the structure and morphology and thermal, rheological, and 
mechanical properties of blends was investigated comprehensively.

\section{Materials and Methods}

2.1. Materials. PLA (PLA711, heat-resistant type) was purchased from Hangzhou Ximao Technology Co., Ltd. (China); the temperature for processing can arrive at $210^{\circ} \mathrm{C}$; PC (PC2407, low viscosity and high impact resistant type) was purchased from Bayer Material Technology Co., Ltd. (German); TPU (85E95W, polyester type) was purchased from Bangtai Chemistry Co., Ltd. (German); di-n-butyltin oxide (DBTO) was purchased from Development of Beijing Chemical Technology Co., Ltd. (China).

2.2. Sample Preparation. The raw materials of PC, PLA, and TPU were dried and the equipment is dewatered before processing in order to avoid the PLA degradation during melt blending. The process of sample preparation is shown as follows: PC, PLA, and TPU were dried in a vacuum oven at $60^{\circ} \mathrm{C}$ for 6 hours, and then PC, PLA, TPU, and DBTO with a certain ratio were put into twin-screw extruder (CTE-35 type, provided by Ningbo Haitian Stock Co., Ltd.) which had been fully dehumidified to melt extrusion and then pelletized, the temperature and screw speed of the extruder were set at $210^{\circ} \mathrm{C}-230^{\circ} \mathrm{C}$ and $120 \mathrm{rpm}$, and the extrusion process should be under vacuum. As has been proved by viscosity and GPC measure, the molecular weight of PLA after extrusion in actual processing temperature decreased within $8 \%$. After being pelletized, the blends were put into the injection molding machine (HTF90WE type, provided by Ningbo Haitian Stock Co., Ltd.) for preparing the samples.

2.3. Characterization. Morphology of blends was observed by scanning electron microscope (SEM) (S-3000N, provided by Hitachi Co., Ltd.), and the samples for observation were the surface and the cross section slices of impact broken blends, respectively. Thermal properties were represented by differential scanning calorimetry instrument (Q20 type, provided by TA Instruments in United States), and its analytical method was as follows: heat from $20^{\circ} \mathrm{C}$ to $200^{\circ} \mathrm{C}$ at the heating rate of $10^{\circ} \mathrm{C} / \mathrm{min}$, keep $3 \mathrm{~min}$ to eliminate thermal history, and then cool it down to room temperature at the rate of $10^{\circ} \mathrm{C} / \mathrm{min}$ and heat it to $200^{\circ} \mathrm{C}$ at the rate of $10^{\circ} \mathrm{C} / \mathrm{min}$ for the second time; the heating process was in nitrogen atmosphere. Thermal stability was represented by thermal gravimetric analyzer (Q50, provided by TA Instruments Company of America), and its analytical method was as follows: heat from $25^{\circ} \mathrm{C}$ to $700^{\circ} \mathrm{C}$ at the rate of $10^{\circ} \mathrm{C} / \mathrm{min}$ in nitrogen atmosphere. Rheological behavior was represented by the capillary rheometer (RH7-D type, provided by Malvern, UK, with die $L / D=16$ ). Tensile strength and elongation at break were tested by the universal mechanical tester (provided by SUNS Company, Shenzhen). Notched impact strength was tested by impact tester (CJJ provided by Zuoji Instruments and Equipment Co., Ltd., Shanghai).

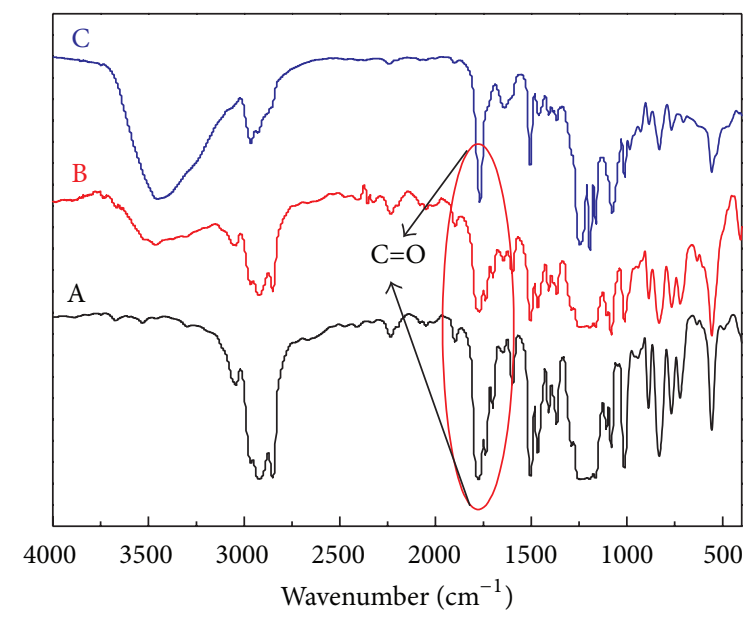

FIgUre 1: Spectrums of PC/PLA/TPU blends (A: PC/PLA/TPU (70/30/10); B: PC/PLA/TPU (70/30/30); C: PC/PLA/TPU/DBTO $(70 / 30 / 30 / 0.8))$.

\section{Result and Discussions}

3.1. Structure and Morphology of Blends. For confirming the occurrence of transesterification, FTIR spectra of PC/PLA/ EVA blends and PC/PLA/EVA blends with catalyst DBTO were presented in Figure 1. As shown in Figure 1, the absorption peaks of both PC/PLA/TPU and PC/PLA/TPU/DBTO blends are similar basically. However, the peaks around $1700 \mathrm{~cm}^{-1}$, corresponding to the $\mathrm{C}=\mathrm{O}$ bond in blend, exhibit obvious distinguishing. The multiple peaks are observed in spectra of PC/PLA/TPU blends with different ratios of raw materials and the separated peaks belong to the $\mathrm{C}=\mathrm{O}$ group of PC, PLA, and TPU in blends, respectively, while, in the spectrum of blend with catalyst DBTO, the corresponding peak changes to be a single peak, revealing the homogenization of these three kinds of $\mathrm{C}=\mathrm{O}$ groups due to the occurrence of transesterification between them.

For analyzing the compatibility of the blends, the surface morphology of blends was shown in Figure 2 for comparison. SEM images (a), (b), (c), and (d) are the surface of blends, respectively. From the images, it could be seen clearly that different sizes of split-phases exist obviously in the surface of PC/PLA blends (image (a)), revealing the obvious poor compatibility. After incorporating TPU, the separated phases still exist, attributed to the unsolved problem related to the compatibility between three components in blends. However, the interface between different phases in blend becomes indistinct, and the addition of TPU may play a function in contacting PLA and PC in some degree. Comparably, the surface of blends added catalyst becomes more smooth without obvious split-phases, indicating that the transesterification indeed improved the compatibility of blend effectively.

3.2. Thermal Properties of Blends. The DSC curves of PLA, PC/PLA, PC/PLA/TPU, and PC/PLA/TPU/DBTO blends were shown in Figure 3. As can be seen in Figure 3, the DSC curve of PLA owns obvious crystallization exothermic peak when the temperature is around $100^{\circ} \mathrm{C}$, and a melting 


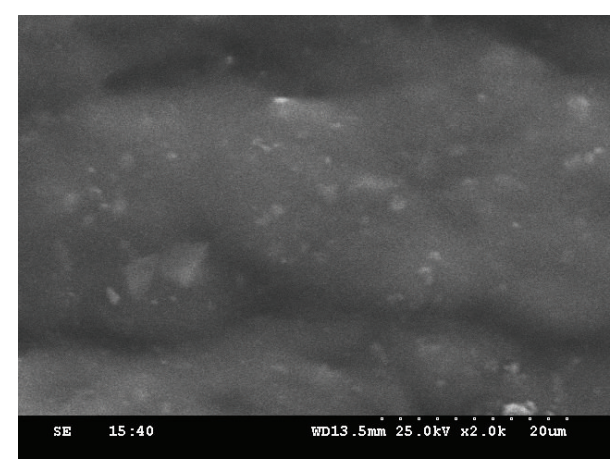

(a)

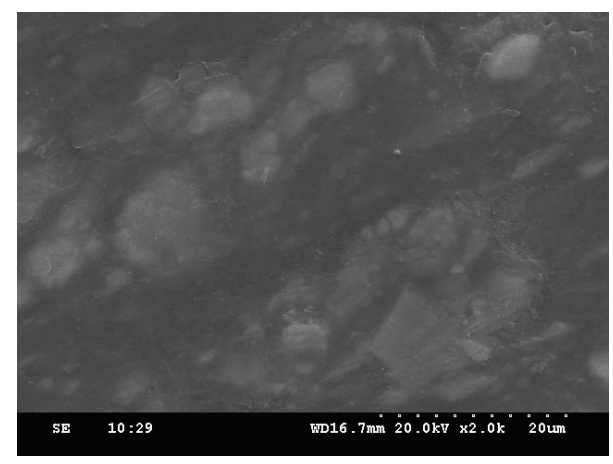

(c)

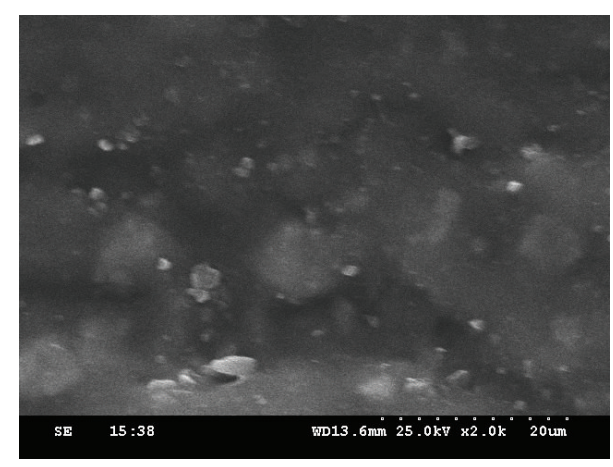

(b)

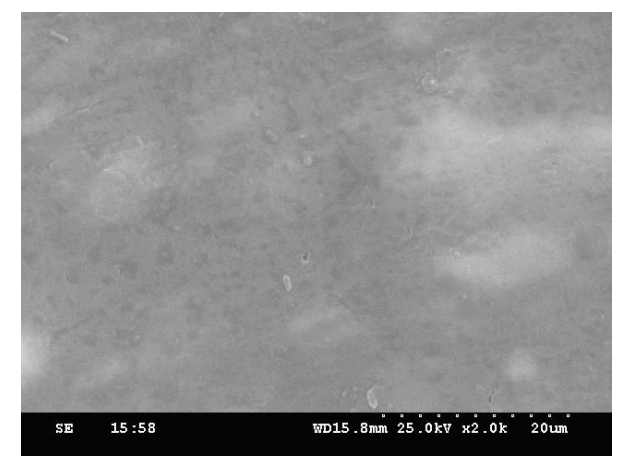

(d)

Figure 2: SEM images of the surface of blends ((a) PC/PLA (70/30); (b) PC/PLA/TPU (70/30/20); (c) PC/PLA/TPU (70/30/40); (d) PC/PLA/TPU/DBTO (70/30/30/0.6)).

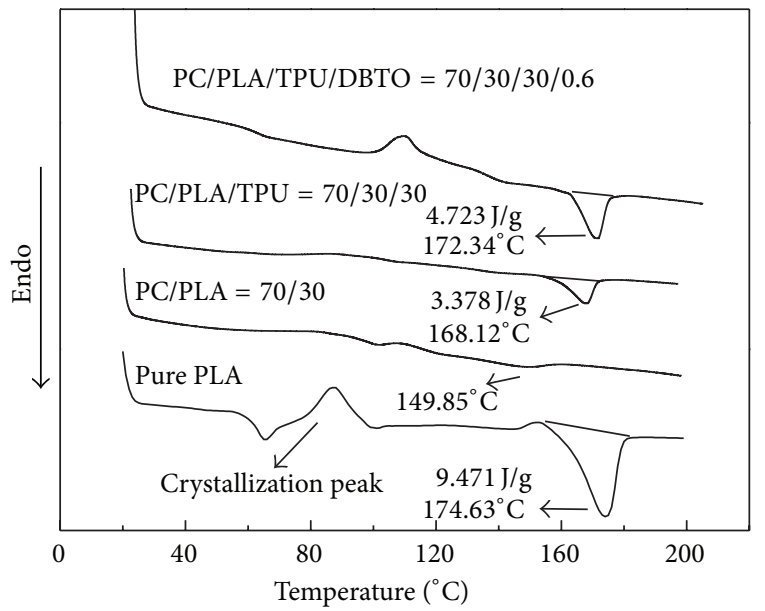

Figure 3: The DSC curves of PLA, PC/PLA, and PC/PLA/TPU blends.

endothermic peak also appears around $170^{\circ} \mathrm{C}$, as a symbol of the crystallization of PLA. In the DSC curve of PC/PLA blend, the glass-transition of $\mathrm{PC}$ appears at $149.83^{\circ} \mathrm{C}$, but there is no obvious melting peak that could be found. The result indicates that the existence of PC in blends impeded the movement and rearrangement of PLA macromolecules chain so as to influence its crystallization. In curves of PC/PLA/TPU and PC/PLA/TPU/DBTO, the melting peaks that appear at $172^{\circ} \mathrm{C}$ and $168^{\circ} \mathrm{C}$ prove the existence of the crystallization of PLA in blends. This may be explained by the idea that the increase of the "soft" chain from TPU decreased the intramolecular and intermolecular force and enhanced the activity and flexibility of PLA molecular chain, which all beneficially affected crystallization of PLA. We can also see that the blend with DBTO shows better ability of crystallizing due to the improvement of compatibility. For displaying the effect of adding TPU and catalyst to the thermal properties of blends more directly, the crystallinity of blends calculated by normalizing the composition of blends is shown in Table 1. The melting enthalpy of PLA with complete crystal is considered $49.3 \mathrm{~J} / \mathrm{g}$. From the data shown in Table 1 , it could be seen that the crystallization of PLA almost disappears in PLA/PC blends, and this may be ascribed to the idea that the molecular chain of PC is so rigid, that is, hard to move, which limits the activity and arrangement of PLA chains. Meantime, with the increase of TPU, the crystallization of PLA appears again and increases. The molecular chains of elastomer TPU own better activity which is conducive to the move of PLA thereby resulting in the increase of crystallization. Moreover, it could also be seen that the addition of DBTO could increase the crystallization further. The transesterification integrates the materials in blend and improves the compatibility and homogeneity of blends; all of this is helpful to the crystallization of PLA.

Figure 4 showed the thermal weight loss curves and derivative curves of PC/PLA, PC/PLA/TPU, and 
TABLE 1: DSC data of PLA and PLA composites.

\begin{tabular}{lccc}
\hline Sample & \multicolumn{4}{c}{$T c c\left({ }^{\circ} \mathrm{C}\right)$} & $\Delta H c(\mathrm{~J} / \mathrm{g})$ & $\mathrm{Xc}(\%)$ \\
\hline PLA & 156.4 & 9.471 & 19.21 \\
PC/PLA $=70: 30$ & - & - & \\
PC/PLA $=70: 40$ & - & - & \\
PC/PLA/TPU $=70: 30: 10$ & 157.1 & 3.152 & 4.31 \\
PC/PLA/TPU $=70: 30: 20$ & 158.7 & 3.341 & 4.98 \\
PC/PLA/TPU $=70: 30: 30$ & 159.1 & 3.378 & 5.45 \\
PC/PLA/TPU $=70: 30: 40$ & 159.8 & 3.381 & 5.88 \\
PC/PLA/TPU/DBTO $=70: 30: 30: 0.4$ & 161.2 & 4.178 & 5.71 \\
PC/PLA/TPU/DBTO = 70:30:30:0.6 & 163.6 & 4.723 & 7.04 \\
PC/PLA/TPU/DBTO $=70: 30: 30: 0.8$ & 164.5 & 4.892 & 7.90 \\
PC/PLA/TPU/DBTO = $70: 30: 30: 1.0$ & 166.3 & 4.792 & 8.33 \\
\hline
\end{tabular}

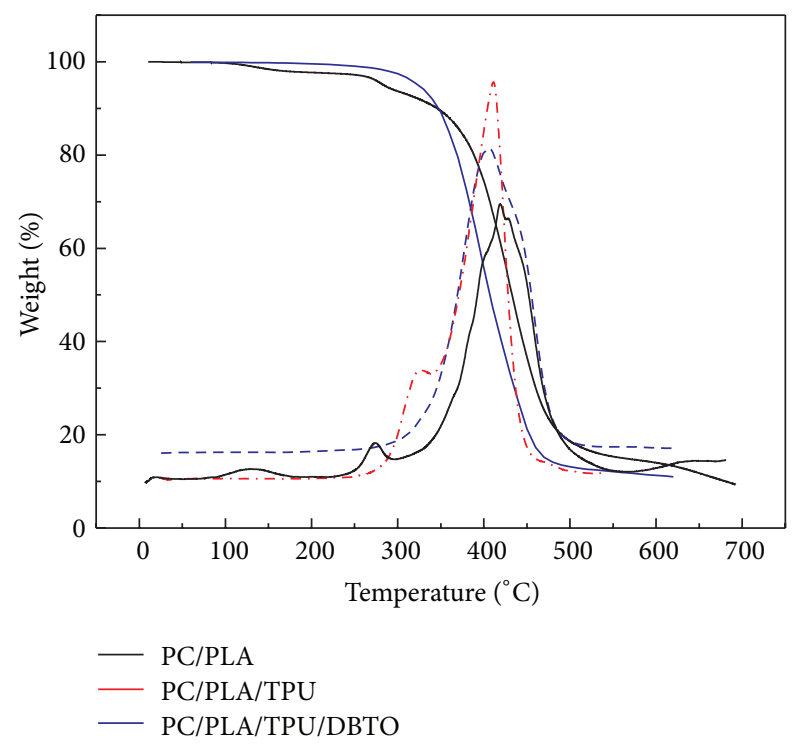

Figure 4: The TGA curves of PC/PLA (70/30), PC/PLA/TPU (70/30/30), and PC/PLA/TPU/DBTO (70/30/30/0.8) blends. The full line: thermogravimetric curve; dash line: derivative thermogravimetry curve.

PC/PLA/TPU/DBTO blends; it is noted that the start temperature for losing weight of PC/PLA is lower than that of PC/PLA/TPU blends, which may be attributed to the reason that TPU chain adsorbed more energy to prohibit the decomposition of PLA, relatively, and the derivative curve of PC/PLA/TPU shifts to low temperature compared to that of PC/PLA blend, indicating that the TPU is against the thermal stability of blends due to the low decomposition temperature of TPU. Moreover, for PC/PLA/TPU/DBTO blends, the curve is similar to that of PC/PLA/TPU curve, while the curve becomes more smooth, corresponding to the more homogeneous loss weight behavior, which also proves the more homogeneous structure of blends.

3.3. Mechanical Properties of Blends. The main purpose of adding elastomer TPU to PC/PLA blends was to improve the toughness of blends. Figure 5 showed the tensile and impact properties of PC/PLA, PC/PLA/TPU, and PC/PLA/TPU/ DBTO blends. It can be seen clearly from Figure 5 (A, B, $\mathrm{C}$, and D series) that the mechanical properties of PC/PLA blends including tensile strength, elongation at break, and impact strength all exhibit a significant deterioration trend with the increase of PLA content, the mechanical data of properties belonging to PC/PLA (70/40) blends is only half of that of pure PC plastic (the impact strength and tensile strength of PC2 2407 are $75 \mathrm{KJ} / \mathrm{m}^{2}$ and $66 \mathrm{MPa}$ ), and the results can be attributed to the low strength of PLA as well as the incompatible phase separation due to the poor compatibility between PLA and PC. After adding TPU, the elongation at break of blends increases significantly with the increase of TPU content, while the tensile strength decreases obviously, revealing that the soft TPU chain is not conducive to the strength but still plays a connection role for PC and PLA, thereby resulting in the improvement of toughness due to the more effective dispersion of energy. Moreover, the impact strength exhibits a first rise and then fall trend; when the content of TPU exceeds 25 copies, the impact strength of blend decreases rapidly. Although the TPU owns good flexibility and could modify the compatibility of blends, its intrinsic strength is not high and high content should cause the occurrence of split phase. More importantly, comparing E curves with D curves in the figure, it could be found that the addition of catalyst improves the mechanical properties significantly, the tensile, and impact strength, and elongation at break increases considerably due to the improvement of the compatibility of blends. Meantime, the properties still show direct dependency on the content of TPU, and the rule is consistent with that of PC/PLA/TPU blends without DBTO.

In order to illuminate the mechanism of property change of blend, the morphology of cross section of blends after impact fracture was observed. In Figure 6, the SEM images of cross section show different break morphologies, revealing the different break behavior. Apparently, the cross section of PC/PLA blends is multilayer with smooth surface without plastic deformation, which is the characteristic belonging to brittle fracture. After incorporating TPU into blends, the fracture-surface displays complex surface morphology instead of obvious multilayer structure and clear substrate deformation, and many plastic deformations appear. This phenomenon may be reasonably explained by the idea that the rubber phases were pulled out from the substrate and absorbed a lot of energy when suffering the impact. The fracture-surface of blend with catalyst exhibits more complex and rough morphology, there are lot of slippages, corrugations, and deformations appears in the edge of fracture section.

3.4. Rheological Properties of Blends. Rheological properties of the blends play a guiding role and make a great effect on processing, which should be paid the attention in preparation of blends. The influence of TPU and catalyst on the rheological properties of PC/PLA/TPU blends was studied. Figure 7 shows the shear rate-apparent viscosity curves and shear rate-shear stress curves of PC/PLA/TPU blends, respectively. As shown in Figure 7(a), the addition of 


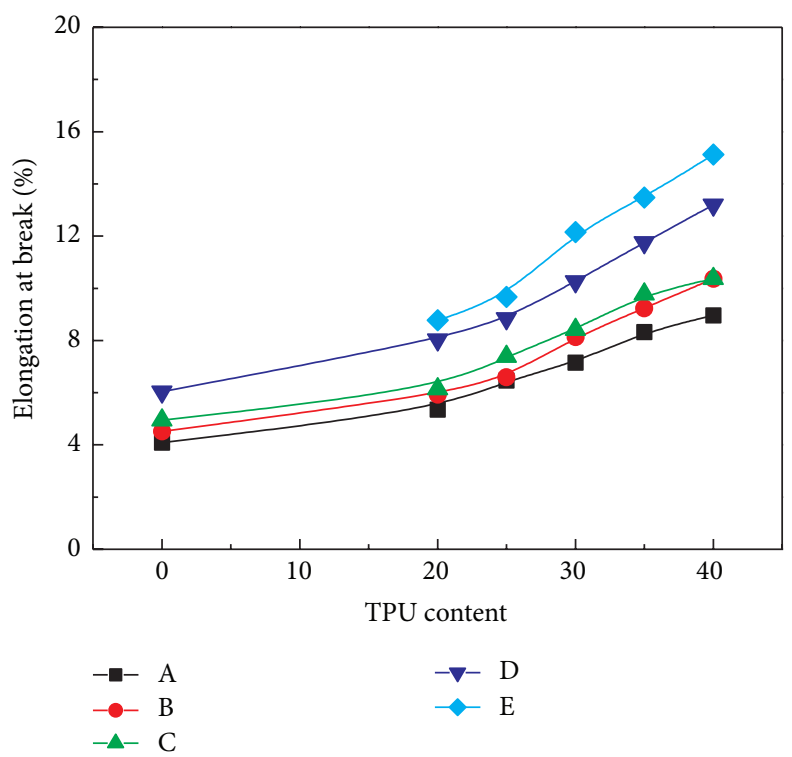

(a)

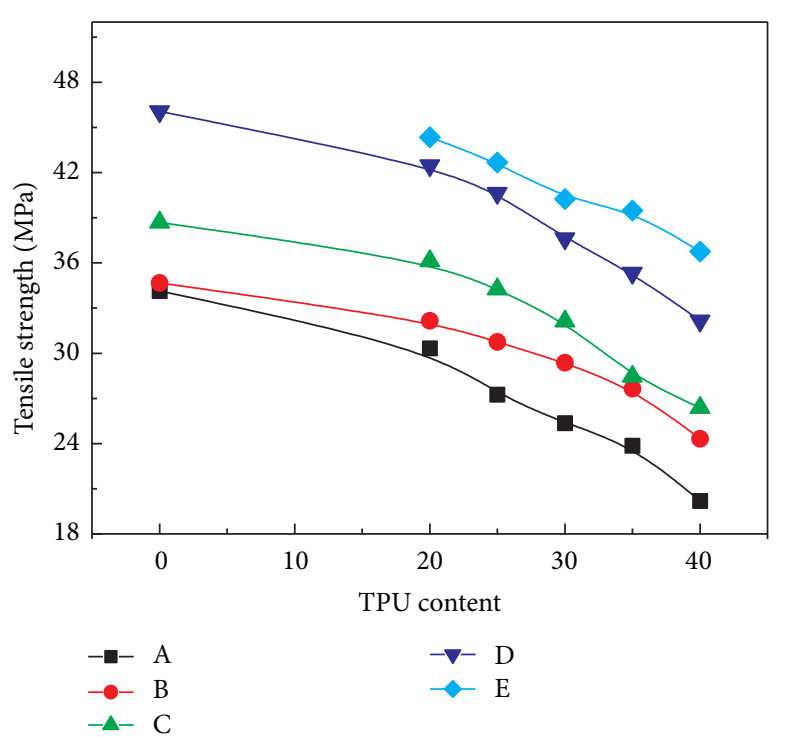

(b)

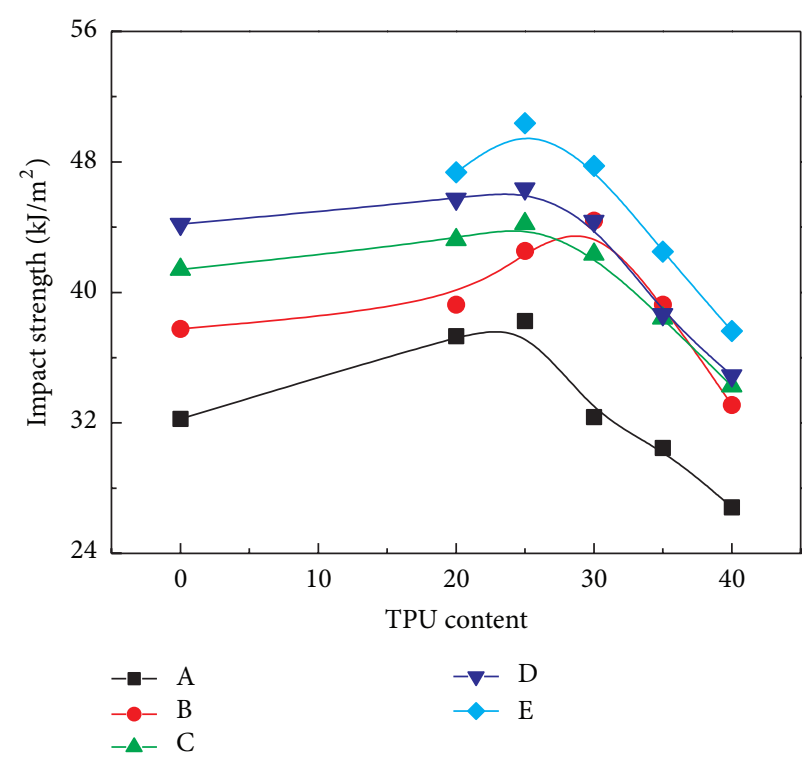

(c)

Figure 5: Mechanical properties of PC/PLA/TPU blends (A: PC/PLA = 50/50; B: PC/PLA = 60/40; C: PC/PLA = 65/35; D: PC/PLA = 70/30; E: $\mathrm{PC} / \mathrm{PLA} / \mathrm{DBTO}=70 / 30 / 0.8$; TPU content is the ratio of copies, e.g., in image (a); TPU content $=30$ corresponds to $\mathrm{PC} / \mathrm{PLA} / \mathrm{TPU}=50 / 50 / 30$ (mass ratio)).

TPU increases the viscosity of blends, which may be ascribed to the higher viscosity of TPU than that of PLA under the processing temperature. However, the range of increase is still limited. At the same time, the addition of catalyst has no obvious influence on the apparent viscosity of blends. Correspondingly, the shear stress of blends increases with increasing TPU content. The non-Newtonian index of blends fitted by shear rate-shear stress curves of blends (Figure 7(b)) was shown in Table 2 , and the non-Newtonian index $n$ of blends comes down with the increase of TPU, indicating that the characteristic of pseudoplasticity of melts becomes more obvious. These phenomena state that the addition of
TPU makes the blends more sensitive to high shear speed. Therefore, increasing the rotate speed of screw is helpful to the processing.

\section{Conclusion}

For developing new materials with high performance and biodegradability, blending engineering plastics PC with biodegradable PLA is an effective method. Though the blends show a higher strength, the significant brittleness is still an obvious defect, attributed to the inherent character of both PC and PLA resin and the poor compatibility between them 


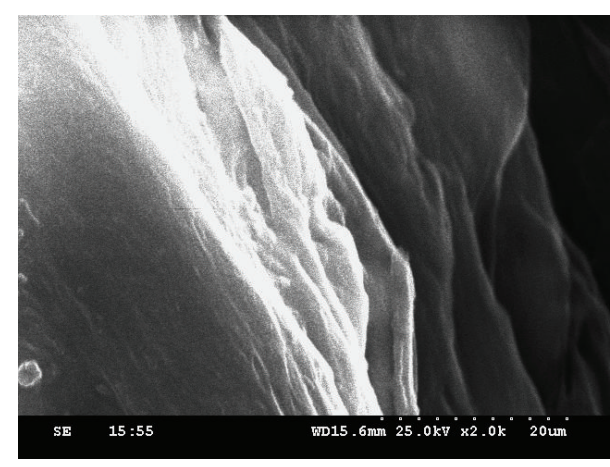

(a)

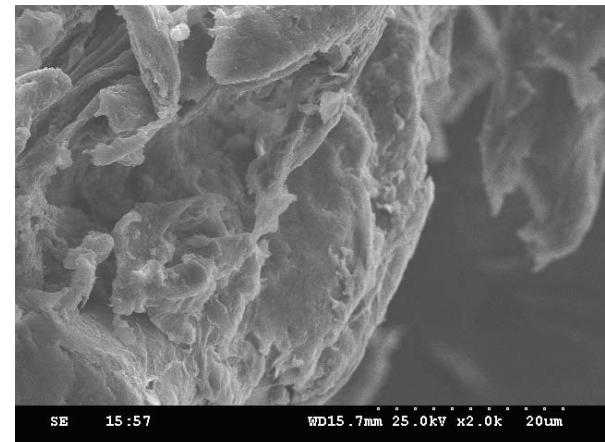

(b)

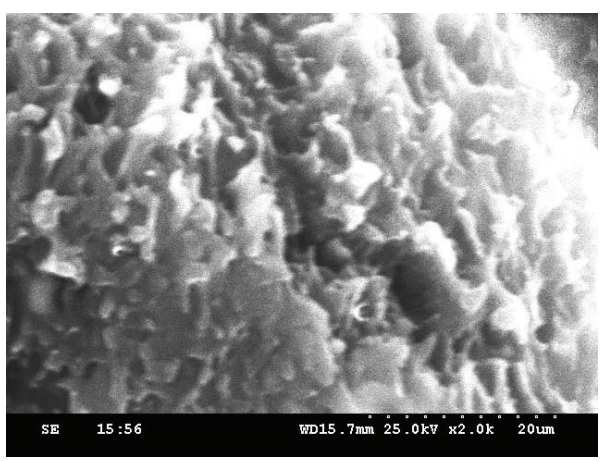

(c)

FIGURE 6: SEM images of the impact break section of blends: (a) PC/PLA (70/30); (b) PC/PLA/TPU (70/30/30); (c) PC/PLA/TPU/DBTO $(70 / 30 / 30 / 0.8)$.

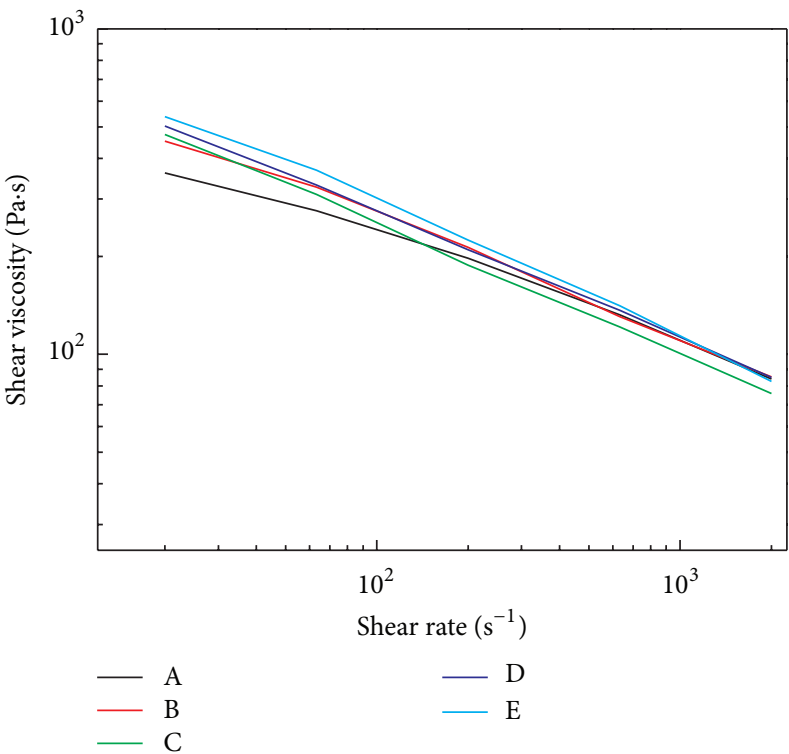

(a)

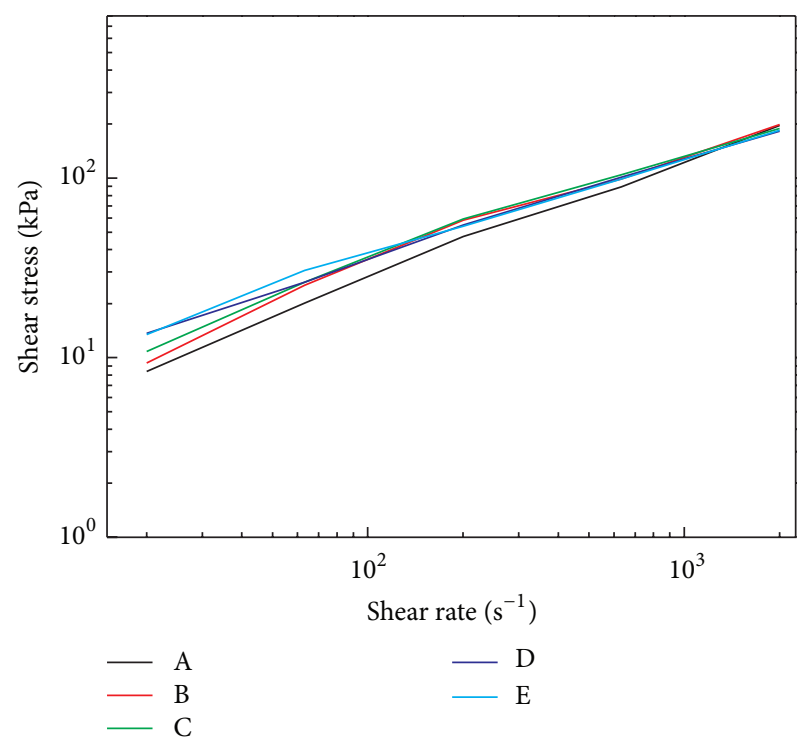

(b)

FIGURE 7: The shear rates-shear viscosity and shear rates-shear stress curves of blends (A: PC/PLA = 70/30; B: PC/PLA/TPU = 70/30/10; C: PC/PLA/TPU = 70/30/20; D: PC/PLA/TPU = 70/30/30; E: PC/PLA/TPU/DBTO = 70/30/30/0.8).

in blends. For solving this problem, TPU, as a toughening modifier, was incorporated into the PC/PLA blends by melt coextrusion. In order to improve the compatibility of blends further, catalyst, DBTO, was also added to blends to catalyze the transesterification of the components in blends. Through investigating the mechanical, thermal, and rheological properties of blends systematically, the several main results were obtained. 
TABLE 2: The non-Newtonian index of blends in Figure 7.

\begin{tabular}{lccccc}
\hline Blends $/ n$ index & A & B & C & D & E \\
\hline Samples in Figure 7 & 0.6768 & 0.6492 & 0.6167 & 0.5674 & 0.5562 \\
\hline
\end{tabular}

Firstly, the addition of TPU improves the toughness of PC/PLA blends significantly, with the increase of TPU, the elongation at break increases considerably, and the impact strength increases firstly and then falls, while the tensile strength decreases significantly. From the observation by SEM, the break section of PC/PLA/TPU blends exhibits typical plastic fracture characteristic. Obviously, the addition of soft TPU chains provides the blends with more flexibility, while greater flexibility caused by high content of TPU damages the strength of blends drastically.

Secondly, with the increase of TPU, the crystallinity of PLA in blends increases gradually and the soft TPU chains decrease the intramolecular and intermolecular force and enhance the activity and flexibility of PLA molecular chains, which is conducive to the arrangement of molecular chains, thereby resulting in a higher crystallinity. Nevertheless, the thermal stability of blends deteriorates slightly.

Thirdly, the apparent viscosity of blends melt decreases with the increase of TPU due to the well melt fluidity of TPU and the melt is closer to the pseudoplasticity melt, which is more sensitive to high shear speed.

Finally, DBTO could catalyze the transesterification of the components in blends effectively, and the structure of PC/PLA/TPU blends becomes more homogeneous after the reaction, revealing the better compatibility. When adding DBTO, the mechanical properties including tensile, impact strength, and elongation at break of blends all increase and the crystallinity of PLA and thermal stability of blends improves. In conclusion, the transesterification catalyzed by DBTO is conducive to the properties of blends. Considering the properties of blends comprehensively, the optimal ratio of PC/PLA/TPU/DBTO is $70: 30: 30: 0.8$.

\section{Conflict of Interests}

The authors declare that there is no conflict of interests regarding the publication of this paper.

\section{Acknowledgments}

The authors acknowledge the financial support of National Science-Technology Support Plan Projects (2014BAD02B06, 2013BAJ10B14), Hunan Province Natural Science Foundation (2015JJ4021, 13JJ1024), Production-Study-Research Cooperation Project of Zhongshan (2013C2FC0022), Science and Technology Planning Project of Guangdong (2013B090600120), and Outstanding Youth Fund of Hunan Province Department of Education (15B064).

\section{References}

[1] H. Zhou, A. H. Touny, and S. B. Bhaduri, "Fabrication of novel PLA/CDHA bionanocomposite fibers for tissue engineering applications via electrospinning," Journal of Materials Science: Materials in Medicine, vol. 22, no. 5, pp. 1183-1193, 2011.

[2] S. Sheng, F. Wang, Q. Ma, and X. Hu, "Impact of foaming air on melting and crystallization behaviors of microporous PLA scaffolds," Journal of Thermal Analysis and Calorimetry, pp. 112, 2015.

[3] J.-Y. Park and I.-H. Lee, "Controlled release of ketoprofen from electrospun porous polylactic acid (PLA) nanofibers," Journal of Polymer Research, vol. 18, no. 6, pp. 1287-1291, 2011.

[4] G. Colomines, S. Domenek, V. Ducruet, and A. Guinault, "Influences of the crystallisation rate on thermal and barrier properties of polylactide acid (PLA) food packaging films," International Journal of Material Forming, vol. 1, supplement 1 , pp. 607-610, 2008.

[5] D. González, A. R. Campos, C. A. M. Cunha, V. Santos, and J. C. Parajó, "Manufacture of fibrous reinforcements for biodegradable biocomposites from Citysus scoparius," Journal of Chemical Technology and Biotechnology, vol. 86, no. 4, pp. 575583,2011

[6] H. Li and M. A. Huneault, "Effect of nucleation and plasticization on the crystallization of poly(lactic acid)," Polymer, vol. 48, no. 23, pp. 6855-6866, 2007.

[7] H. Wang, X. Z. Sun, and P. Seib, "Mechanical properties of poly(lactic acid) and wheat starch blends with methylenediphenyl diisocyanate," Journal of Applied Polymer Science, vol. 84, no. 6, pp. 1257-1262, 2002.

[8] N. Wang, J. G. Yu, P. R. Chang, and X. Ma, "Influence of formamide and water on the properties of thermoplastic starch/poly(lactic acid) blends," Carbohydrate Polymers, vol. 71, no. 1, pp. 109-118, 2008.

[9] C.-S. Wu, "Improving polylactide/starch biocomposites by grafting polylactide with acrylic acid-characterization and biodegradability assessment," Macromolecular Bioscience, vol. 5, no. 4, pp. 352-361, 2005.

[10] T. K. Ke and X. S. Sun, "Starch, Poly(lactic acid), and Poly(vinyl alcohol) Blends," Journal of Polymers and the Environment, vol. 11, no. 1, pp. 7-14, 2003.

[11] T. Takayama and M. Todo, "Improvement of impact fracture properties of PLA/PCL polymer blend due to LTI addition," Journal of Materials Science, vol. 41, no. 15, pp. 4989-4992, 2006.

[12] W. S. Chow and S. K. Lok, "Thermal properties of poly(lactic acid)/organo-montmorillonite nanocomposites," Journal of Thermal Analysis and Calorimetry, vol. 95, no. 2, pp. 627-632, 2009.

[13] N. W. Zhang, Q. F. Wang, J. Ren, and L. Wang, "Preparation and properties of biodegradable poly(lactic acid)/poly(butylene adipate-co-terephthalate) blend with glycidyl methacrylate as reactive processing agent," Journal of Materials Science, vol. 44, no. 1, pp. 250-256, 2009.

[14] B. Suksut and C. Deeprasertkul, "Effect of nucleating agents on physical properties of poly(lactic acid) and its blend with natural rubber," Journal of Polymers and the Environment, vol. 19, no. 1, pp. 288-296, 2011.

[15] W. Zhang, L. Chen, and Y. Zhang, "Surprising shape-memory effect of polylactide resulted from toughening by polyamide elastomer," Polymer, vol. 50, no. 5, pp. 1311-1315, 2009.

[16] T. Seelig and E. Van der Giessen, "Effects of microstructure on crack tip fields and fracture toughness in PC/ABS polymer blends," International Journal of Fracture, vol. 145, no. 3, pp. 205222, 2007. 
[17] Y. Zhao, Y.-X. Liao, B. Yin, and M.-B. Yang, "Study on the reaction of $\mathrm{PC}$ and EVA during processing," Polymeric Materials Science and Engineering, vol. 21, no. 6, pp. 201-208, 2005.

[18] Q. Sun, C. R. Zheng, S. Wu, S. Cheng, and X. C. Dai, "The study of PC modified by TPU," Polymer Materials Science \& Engineering, vol. 15, pp. 140-144, 1999.

[19] R.-L. Yu, L.-S. Zhang, Y.-H. Feng, R.-Y. Zhang, and J. Zhu, "Improvement in toughness of polylactide by melt blending with bio-based poly(ester)urethane," Chinese Journal of Polymer Science, vol. 32, no. 8, pp. 1099-1110, 2014.

[20] R. Al-Itry, K. Lamnawar, A. Maazouz, N. Billon, and C. Combeaud, "Effect of the simultaneous biaxial stretching on the structural and mechanical properties of PLA, PBAT and their blends at rubbery state," European Polymer Journal, vol. 68, pp. 288-301, 2015.

[21] R. Al-Itry, K. Lamnawar, and A. Maazouz, "Biopolymer blends based on poly (lactic acid): shear and elongation rheology/structure/blowing process relationships," Polymers, vol. 7, no. 5, pp. 939-962, 2015.

[22] B. Mallet, K. Lamnawar, and A. Maazouz, "Improvement of blown film extrusion of poly (lactic acid): structure-processingproperties relationships," Polymer Engineering \& Science, vol. 54, pp. 840-857, 2014.

[23] R. Al-Itry, K. Lamnawar, and A. Maazouz, "Rheological, morphological, and interfacial properties of compatibilized PLA/PBAT blends," Rheologica Acta, vol. 53, no. 7, pp. 501-517, 2014.

[24] R. Al-Itry, K. Lamnawar, and A. Maazouz, "Reactive extrusion of PLA, PBAT with a multi-functional epoxide: physicochemical and rheological properties," European Polymer Journal, vol. 58, pp. 90-102, 2014.

[25] R. Al-Itry, K. Lamnawar, and A. Maazouz, "Improvement of thermal stability, rheological and mechanical properties of PLA, PBAT and their blends by reactive extrusion with functionalized epoxy," Polymer Degradation and Stability, vol. 97, no. 10, pp. 1898-1914, 2012. 

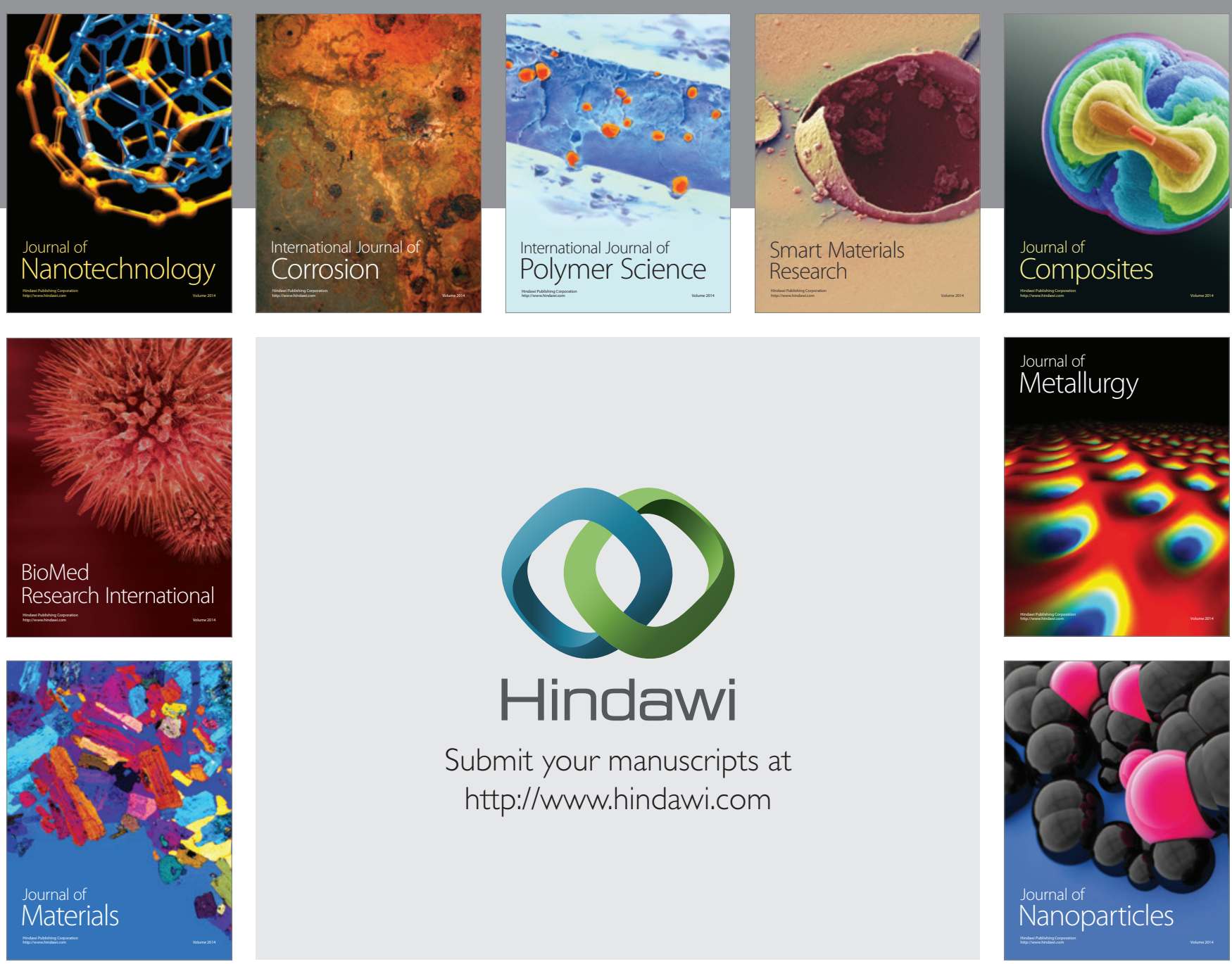

Submit your manuscripts at http://www.hindawi.com
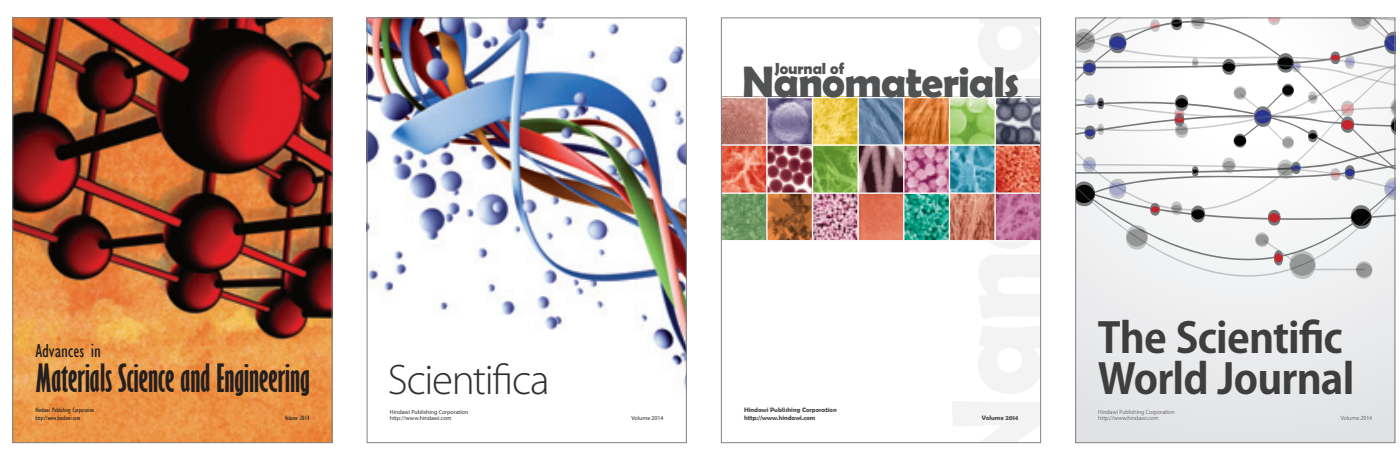

\section{The Scientific World Journal}
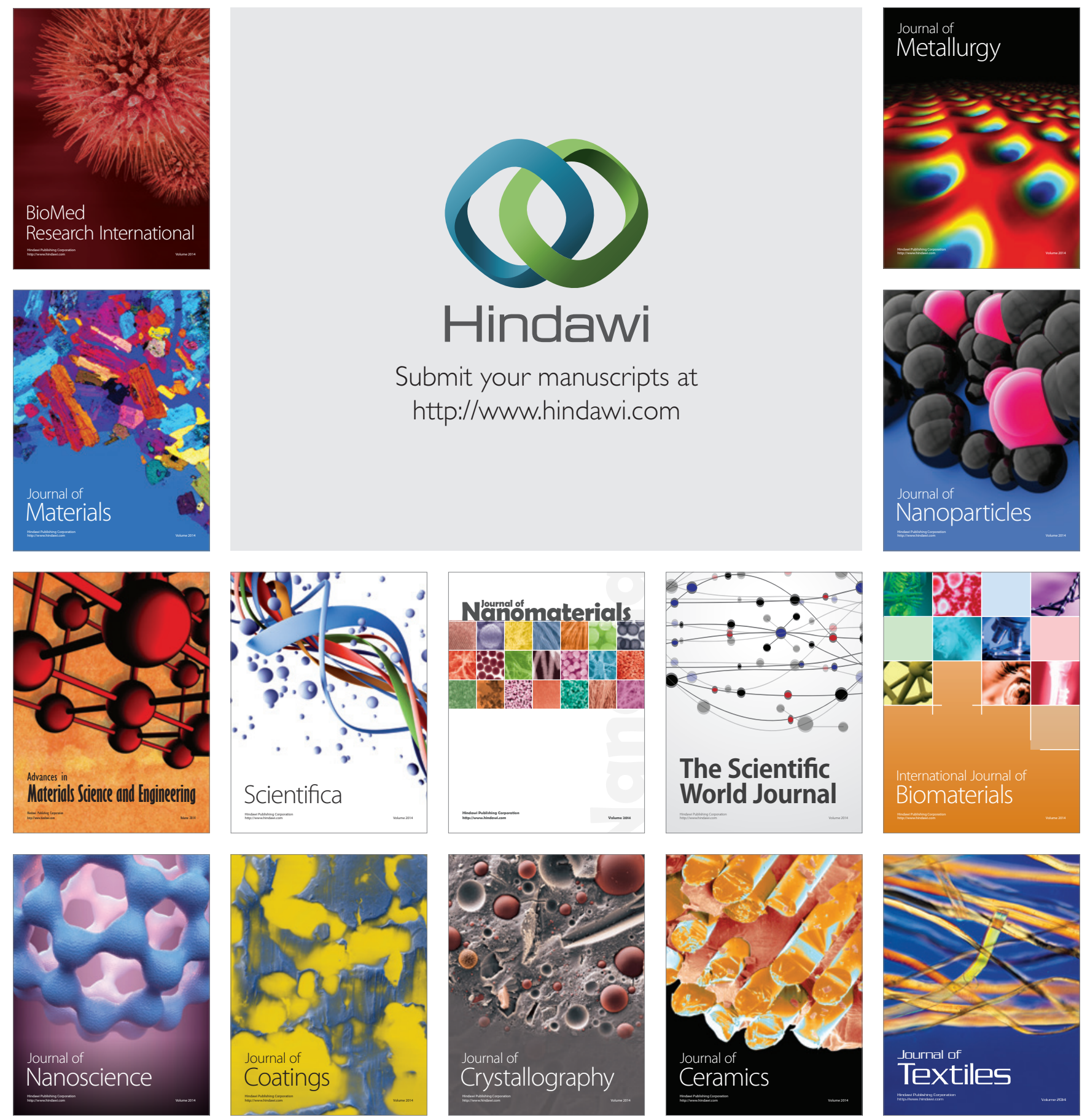